\title{
A ATUAL CLASSIFICAÇÃO DO ANTIGO GÊNERO TABEBUIA (BIGNONIACEAE), SOB O PONTO DE VISTA DA ANATOMIA DA MADEIRA ${ }^{1}$
}

\author{
SIDINEI RODRIGUES DOS SANTOS ${ }^{2}$
}

\section{RESUMO}

O gênero Tabebuia (Bignoniaceae) sofreu inúmeras alterações ao longo de sua história, sendo recentemente dividido em três novos gêneros: Tabebuia, Handroanthus e Roseodendron. O presente estudo teve como objetivo verificar se essas modificações também se refletem na anatomia da madeira. Foram analisados caracteres anatômicos de vasos, raios e fibras de 43 espécies pertencentes aos três gêneros, retirados da literatura. Os resultados depõem favoravelmente à segregação dos novos gêneros, conforme sugerido em estudos taxonômicos recentes. Apesar de não haver uma característica ou um conjunto de características anatômicas comuns e exclusivas às espécies de cada um dos gêneros, como atualmente circunscritos, é inegável que distintas tendências estruturais justificam a separação dos mesmos.

Palavras-chave: Tabebuia, Handroanthus, Roseodendron, Ipê.

\section{ABSTRACT}

[The new classification of the former genus Tabebuia (Bignoniaceae), according to wood anatomy]. The genus Tabebuia (Bignoniaceae) has experienced numerous taxonomic changes throughout its history, being recently divided in three new genera: Tabebuia, Handroanthus and Roseodendron. The present work aimed to verify if these changes are also reflected in the wood anatomy. Anatomical characters of vessels, rays and fibers of 43 species belonging to the three genera were taken from literature. The results support the seggregation of the new genera, as suggested in recent taxonomic studies. Despite the absence of an anatomical feature or a group of anatomical features that are common (and exclusive) among species of each new genera, as currently circumscribed, it is undeniable that distinct structural tendencies can be recognized, justifying the actual seggregation.

Key words: Tabebuia, Handroanthus, Roseodendron, Ipê.

\section{INTRODUÇÃO}

Pertencente à família Bignoniaceae, o gênero Tabebuia constitui um importante grupo de plantas neotropicais, distribuídas desde o sudoeste dos Estados Unidos até o norte da Argentina e Chile. Trata-se do maior gênero da família, totalizando cerca de 100 espécies, muitas delas amplamente reconhecidas pelo valor ornamental e qualidade das madeiras que produzem (Grose e Olmstead, 2007b). O Brasil é um dos países mais bem representados, sendo encontradas, aproximadamente, 40 espécies de "ipês" (FloradoBrasil).

Complexo, do ponto de vista taxonômico, o gênero sofreu inúmeras alterações ao longo de

1 Recebido para publicação em 10-03-2017 e aceito para publicação em 07-06-2017.

2 Biólogo, Dr., Universidade Federal do Pampa. Dom Pedrito, RS, Brasil.sthurt.bio@gmail.com sua história. Originalmente, todas as espécies conhecidas como ipês eram incluídas no gênero Tabebuia, o qual foi criado por De Candolle (1838) para abrigar as Bignoniáceas arbóreas de folhas simples. A descrição de novas espécies na família e a grande variabilidade morfológica com que se apresentavam, no entanto, acabaram levando a uma expansão nos limites genéricos iniciais. Assim, à medida que surgiam novos estudos, o conceito tradicional se modificava, de modo que a circunscrição original não se manteve ao longo do tempo. As modificações ocorridas incluíram a sinonimização, segregação e reincorporação de inúmeras espécies, inclusive de outros gêneros, como Tecoma, provocando uma grande confusão no grupo taxonômico em questão (Grose e Olmstead, 2007a,b).

Ciente da heterogeneidade existente no grupo - e procurando resolver este problema -, 
Mattos (1970) dividiu as espécies de ipês em dois gêneros, Tabebuia e Handroanthus, este último criado, especialmente, para os representantes brasileiros. Tal interpretação foi fortemente criticada por Gentry (1972; 1992), que tornou a posicionar todas as espécies de ipês no grupo original. Mais recentemente, Grose e Olmstead (2007b) propuseram, com base em estudos filogenéticos, a divisão de Tabebuia em três gêneros, Tabebuia, Handroanthus e Roseodendron, confirmando, em definitivo, a segregação proposta por Mattos (1970) e a existência de linhagens diferentes dentro do grupo.

Por refletir, em tese, a verdadeira relação de parentesco entre as espécies, esta é, atualmente, a classificação genérica de Tabebuia mais aceita pela comunidade científica. Tendo em vista as recentes alterações, o presente estudo pretende avaliar se as diferenças apontadas pela análise morfológica e molecular também se refletem na anatomia da madeira dos três novos gêneros, do modo como estão circunscritos.

\section{REVISÃO DA LITERATURA}

Apesar da importância, são poucos os estudos que tratam das relações internas de Tabebuia. Um dos primeiros trabalhos envolvendo o gênero dos ipês e a família Bignoniaceae como um todo foi realizado por Record \& Hess (1943). Nele, os autores fornecem uma caracterização geral da família, incluindo detalhes anatômicos. Com relação ao gênero em questão, foram reconhecidos quatro grupos de espécies, incluindo o grupo "lapacho", o maior e mais importante deles, caracterizado pela alta densidade da madeira, que tem cor marrom-esverdeada e abundantes conteúdos amarelos no cerne. Esse conjunto de espécies que forma o grupo lapacho, no entanto, já era conhecido por Record \& Hess desde 1940, pelo menos, ano da publicação de American Timbers of the Family Bignoniaceae, onde já se fazia menção a sua existência.

Outro trabalho que apontou diferenças relevantes na anatomia dos ipês foi realizado por Santos e Miller (1992). Nesse estudo, foram reconhecidos três grupos de espécies, separados por diferenças no tamanho das pontoações intervasculares, espessura da parede de fibras, largura e estratificação de raios, bem como pela densidade e presença (ou não) de conteúdos amarelados na madeira (lapachol). O Grupo I, caracteriza-se pela alta densidade da madeira $\left(>0,74 \mathrm{~g} / \mathrm{cm}^{3}\right)$, pelo cerne muito distinto do alburno, marrom-esverdeado a enegrecido, com bastante conteúdo amarelado nos vasos (lapachol), bem como pela presença de pontoações intervasculares grandes $(8-14 \mu \mathrm{m})$, fibras de paredes muito espessas e raios com estrutura estratificada bem definida. O Grupo II, caracteriza-se pela madeira de média densidade $\left(0,4-0,74 \mathrm{~g} / \mathrm{cm}^{3}\right)$, com cerne castanhoclaro a avermelhado, pouco distinto do alburno e sem lapachol, pelas pontoações intervasculares pequenas $(3-6 \mu \mathrm{m})$, pelas fibras de paredes finas a espessas e pelos raios irregularmente estratificados ou não estratificados, com 1-2 células de largura. O Grupo III, não muito distinto do Grupo II em aspectos macro e microscópicos, caracteriza-se pela alta variabilidade intraespecífica das madeiras.

Além desses três grupos, os autores identificaram um conjunto de quatro espécies que não se enquadram em nenhum dos grupos anteriores: Tabebuia chrysea, Tabebuia donnellsmithii, Tabebuia fluviatilis e Tabebuia nodosa. Tabebuia chrysea, embora com presença de lapachol nos vasos, não foi incluída no grupo I por apresentar algumas características típicas de outros grupos, como madeira de densidade média, raios com estratificação ausente ou irregular e pontoações intervasculares pequenas. Tabebuia donnell-smithii foi separada, principalmente, pela largura excepcional dos raios (45 , até 8 células). Tabebuia nodosa, apesar de apresentar estrutura estratificada, fibras de paredes muito espessas e raios com 2-3 células de largura, também não foi incluída no grupo I, sobretudo pelo parênquima vasicêntrico e tamanho reduzido das pontoações intervasculares. Tabebuia fluviatilis, como Tabebuia 
donnell-smithii, não diferiu muito das espécies dos grupos II e III, separando-se, todavia, devido à peculiaridade na altura dos raios.

O trabalho mais recente envolvendo anatomia da madeira e questões taxonômicas de Tabebuia é de Pace et al. (2015), no qual são investigados vários gêneros das principais linhagens da família Bignoniaceae, incluindo 7 espécies de Tabebuia, 5 de Handroanthus e 1 de Roseodendron. Os autores concluíram que a nova circunscrição taxonômica de Tabebuia, proposta por Grose e Olmstead (2007a), é fortemente suportada pela anatomia. Segundo os autores, o grupo "Lapacho", proposto por Record e Hess (1943), corresponde ao Grupo I de Santos e Miller (1992), o qual coincide, perfeitamente, com Handroanthus. Microscopicamente, este gênero se diferencia pela presença de pontoações intervasculares grandes $(>10 \mu \mathrm{m})$, fibras de paredes muito espessas e raios estratificados, com 2-3 células de largura. As espécies dos Grupos II e III correspondem, por sua vez, a Tabebuia, que é caracterizado por ter pontoações intervasculares pequenas (5-8 $\mu \mathrm{m})$, fibras de paredes finas a espessas e raios uni a bisseriados, com estratificação normal a irregular. Apesar de Roseodendron donnellsmithii ter sido incluído no trabalho, não são se forneceu maiores considerações sobre o gênero, comentando-se, apenas, que Tabebuia chrysea e Tabebuia donnell-smithii incluem-se entre as quatro espécies que não se enquadram nos grupos anatomicamente definidos por Santos e Miller (1992), e que elas integram o novo gênero Roseodendron.

\section{MATERIAIS E MÉTODOS}

No presente estudo de revisão foram investigados detalhes da anatomia da madeira de 43 espécies, nativas e exóticas, pertencentes ao antigo gênero Tabebuia (Bignoniaceae). A relação dos nomes científicos válidos e as respectivas sinonímias, bem como os caracteres anatômicos investigados e respectivos trabalhos de onde foram retirados os dados, encontramse resumidos na Tabela 1. Foram analisados os seguintes aspectos: tamanho das pontoações intervasculares, espessura da parede de fibras, estratificação e largura de raios em número de células, que são algumas das características mais utilizadas para a separação de espécies ou grupo de espécies em Tabebuia (Record e Hess, 1940; Record e Hess, 1943; Santos e Miller, 1992; Pace et al., 2015). Para facilitar a análise e homogeneizar os dados e terminologias, já que se trata de um trabalho de revisão, alguns caracteres foram separados em categorias.

As pontoações foram divididas em duas categorias: pequenas a médias (até $7 \mu \mathrm{m}$ ) e médias a grandes (maiores de $7 \mu \mathrm{m}$ ). No estudo das pontoações, foram usados os valores médios, exceto quando especificado o contrário. Quando se dispõe, apenas, os valores mínimos e máximos, como no caso de Santos e Miller (1992), estes somente foram incluídos na análise se o seu intervalo situa-se, inteiramente, dentro dos limites de cada categoria. Com isso, garantiuse que a média, mesmo sendo conhecida, jamais poderia dar um resultado diferente. Como o tamanho das pontoações na maioria das famílias (incluindo as Bignoniaceae) não costuma apresentar grandes desvios padrões, os valores mínimos e máximos não se afastam muito da média, de modo que este não foi um grande empecilho ao estudo.

Para a espessura da parede das fibras, criaram-se, igualmente, duas categorias, nas quais foram incluídos todos os estados de caráter descritos na literatura consultada: finas a espessas e espessas a muito espessas. As fibras originalmente descritas nos trabalhos como muito finas, finas, ou finas a espessas foram enquadradas na primeira categoria; aquelas caracterizadas como espessas, muito espessas ou espessas a muito espessas, ficaram na segunda.

No caso da estratificação dos raios, além de sua presença (ou não), também foi anotada a sua regularidade (regular ou irregular), resultando, neste caso, três categorias: presente/ regular, presente/irregular e ausente. A largura dos raios não foi separada em classes (Tabela 
1). As fotomicrografias apresentadas para ilustrar detalhes importantes da anatomia foram retiradas dos mesmos trabalhos que deram origem aos dados, sendo indicada a sua referência na legenda.

\section{RESULTADOS E DISCUSSÃO}

A heterogeneidade estrutural em Tabebuia não é nenhuma novidade, e basta, para isso, verificar-se a literatura. A dúvida, como inicialmente salientado, é se essas características (ou conjunto de características), reconhecidamente variáveis nas espécies, apresentam (ou não) uma distribuição compatível com a atual circunscrição do gênero (Grose e Olmstead, 2007b). Essa questão começou a ser respondida por Pace et al. (2015), apesar de o seu estudo não tratar, especificamente, sobre as alterações taxonômicas ocorridas em Tabebuia, mas ainda requer análise mais minuciosa.

Com vistas a confirmar (ou não) se, de fato, a anatomia da madeira difere entre as espécies dos novos gêneros atualmente propostos, reuniuse, da literatura, dados sobre algumas características anatômicas reconhecidamente úteis para a separação de espécies ou grupo de espécies em Tabebuia. Foram analisadas, ao todo, 43 espécies nativas e exóticas pertencentes aos três taxa segregados do antigo gênero Tabebuia (Tabebuia, Handroanthus e Roseodendron). Um resumo dos caracteres anatômicos, espécies e referências utilizadas é fornecido na Tabela 1 .

Se fosse considerado como critério para a separação ou não das espécies do antigo gênero Tabebuia a ocorrência de características anatômicas comuns e exclusivas, isto é, que estão presentes em todos os membros de um grupo, mas ausentes nos demais, como preconizado pela filogenética, estaria descartada a separação, pelo menos do ponto de vista da anatomia da madeira, uma vez que nenhuma das características anatômicas avaliadas se encaixa em tal perfil na atual circunscrição genérica. No entanto, é preciso ter em mente que os estados de caráter em anatomia da madeira, principalmente no caso de características quantitativas, costumam apresentar variações nos indivíduos e, inclusive, dentro de um mesmo indivíduo, por diversos motivos, de modo que separar grupos de espécies em categorias estanques com base nestes critérios é quase sempre uma tarefa bastante difícil, se não impossível, ainda mais quando há um grande número de taxa envolvido. Alguns exemplos de variabilidade na estrutura anatômica em Tabebuia podem ser encontrados nos trabalhos de Carrera (2008) e Doria (2014).

De fato, muitas das espécies investigadas apresentam características anatômicas variáveis, e basta uma rápida passada de olhos na Tabela 1 para confirmar-se o fato. Mesmo assim, é inegável que existem tendências anatômicas divergentes entre os grupos de espécies que compõem os novos gêneros, concordando-se, neste aspecto, com os resultados de Pace et al. (2015), bem como com o sugerido pela análise molecular (Grose e Olmstead, 2007a). Não é um trabalho simples, todavia, sistematizar, nos gêneros as características anatômicas observadas, dada a variabilidade com que se apresentam. Se a existência de variação intraespecífica dificulta a circunscrição dos gêneros, ela atrapalha e confunde ainda mais a identificação das espécies, motivo pelo qual, em muitos casos, pode ser mais fácil (ou até mais recomendável) o reconhecimento de determinado taxa como pertencente a um ou outro gênero, do que a essa ou aquela espécie.

Como se pode observar na Tabela 1 , todas as espécies atualmente pertencentes a Tabebuia apresentam pontoações intervasculares diminutas a pequenas (até $7 \mu \mathrm{m}$ ), com exceção de Tabebuia fluviatilis, que também pode apresentar pontoações médias a grandes (>7 $\mu \mathrm{m})$. Em Handroanthus, observa-se uma tendência mais ou menos oposta, isto é, as espécies, neste gênero, costumam desenvolver pontoações intervasculares médias a grandes, embora também possam apresentar, não raro, pontoações diminutas a pequenas. Pode-se estabelecer, de maneira mais simples, que o 
TABELA 1 - Espécies, características anatômicas, valores e referências.

\begin{tabular}{|c|c|c|c|c|c|c|c|}
\hline \multirow[t]{2}{*}{ ESPÉCIE } & \multicolumn{3}{|c|}{ PIV } & \multirow{2}{*}{$\begin{array}{c}\mathrm{RL} \\
\text { (cels) }\end{array}$} & \multirow[t]{2}{*}{ RE } & \multicolumn{2}{|c|}{ EPF } \\
\hline & $\varnothing$ & $d p$ & $m g$ & & & fe & me \\
\hline \multicolumn{8}{|l|}{ Tabebuia angustata } \\
\hline 1. Santos e Miller (1992) & $4-6 *$ & $\mathrm{x}$ & & $1-2$ & I & $x$ & \\
\hline 2. Gasson e Dobbins (1991) & 3,3 & $x$ & & $1-2$ & $\mathrm{~N}$ & $x$ & \\
\hline \multicolumn{8}{|l|}{ Tabebuia aurea } \\
\hline 1. Mattos et al. (2003) & & & & $1-3$ & I & & $x$ \\
\hline 2. Dória (2014) & $4,6-5,9$ & $\mathrm{x}$ & & $1-2$ & $N \backslash I$ & $x$ & \\
\hline 3. Yajure (2014) & $3,7-5$ & $x$ & & $1-2$ & A & $x$ & \\
\hline 4. Pace et al. (2015) & 5,2 & $x$ & & 2 & ? & $x$ & \\
\hline 5. Santos e Miller (1992) & $4-6 *$ & $\mathrm{x}$ & & 1 & 1 & $x$ & \\
\hline 6. Gasson e Dobbins (1991) & 4,4 & $x$ & & 1 & 1 & $x$ & \\
\hline 7. Faria (2016) & & & & & $\mathrm{N}$ & $x$ & \\
\hline 8. Alves e Angyalossy- Alfonso (2002) & & & & 1 & & $x$ & \\
\hline 9. Mainieri (1983) & & & & & $\mathrm{N}$ & & \\
\hline 10. Insidewood & 6 & $x$ & & $1-2 \backslash 1-3$ & I\A & $x$ & $x$ \\
\hline \multicolumn{8}{|l|}{ Tabebuia berteroi } \\
\hline 1. Santos e Miller (1992) & $3-4 *$ & $x$ & & $1-2$ & 1 & $x$ & \\
\hline \multicolumn{8}{|l|}{ Tabebuia cassinoides } \\
\hline 1. Barros e Callado (1997) & 3,2 & $\mathrm{x}$ & & 1 & A & $x$ & \\
\hline 2. Kuniyoshi (1993) & & $x$ & & $1-2$ & I & $x$ & \\
\hline 3. Carrera (2008) & $2,3-3,5$ & $x$ & & $1 \backslash 1-2$ & A & $x$ & \\
\hline 4. Pace et al. (2015) & 4,3 & $x$ & & & ? & $x$ & \\
\hline 5. Gasson e Dobbins (1991) & 3,3 & $\mathrm{x}$ & & $1-2$ & A & $x$ & \\
\hline 6. Alves e Angyalossy- Alfonso (2002) & & & & 1 & & $x$ & \\
\hline 7. Mainieri (1983) & & & & & A & & \\
\hline 8. Insidewood & & $x$ & & $1-2 \backslash 1-3$ & I\A & $x$ & \\
\hline \multicolumn{8}{|l|}{ Tabebuia dubia } \\
\hline 1. Santos e Miller (1992) & $5-6 *$ & $x$ & & 1 & 1 & $x$ & \\
\hline \multicolumn{8}{|l|}{ Tabebuia fluviatilis } \\
\hline 1. Pace et al. (2015) & 8,3 & & $x$ & 1 & ? & $x$ & \\
\hline 2. Santos e Miller (1992) & $3-4^{*}$ & $x$ & & $2-3$ & $\mathrm{I}$ & & \\
\hline 3. Insidewood & & & $x$ & $1 \backslash 1-2$ & A & $x$ & \\
\hline \multicolumn{8}{|l|}{ Tabebuia haemantha } \\
\hline 1. Santos e Miller (1992) & $3-5 *$ & $\mathrm{x}$ & & $1-2$ & A & $x$ & \\
\hline 2. Gasson e Dobbins (1991) & 3,9 & $x$ & & $1-2$ & $\mathrm{~N}$ & $x$ & \\
\hline \multicolumn{8}{|l|}{ Tabebuia heterophylla } \\
\hline 1. Pace et al. (2015) & & & & & ? & $x$ & \\
\hline 2. Santos e Miller (1992) & $4-5 *$ & $x$ & & $1-3$ & $N \backslash I \backslash A$ & $x$ & \\
\hline 3. Insidewood & & & & $1-3$ & $N \backslash I$ & $x$ & \\
\hline \multicolumn{8}{|l|}{ Tabebuia insignis } \\
\hline 1. Yajure (2014) & $2,5-5$ & $\mathrm{x}$ & & $1-2$ & $\mathrm{~N}$ & $x$ & \\
\hline 2. Santos e Miller (1992) & $5-6 *$ & $\mathrm{x}$ & & 1 & A & $x$ & \\
\hline 3. Gasson e Dobbins (1991) & 4,1 & $x$ & & $1-2$ & A & $x$ & \\
\hline 4. Miller e Detienne (2001) & $5-6$ & $x$ & & 1 & I\A & $x$ & \\
\hline 5. Alves e Angyalossy- Alfonso (2002) & & & & 1 & & $x$ & \\
\hline
\end{tabular}




\begin{tabular}{|c|c|c|c|c|c|c|}
\hline 6. Insidewood & & $x$ & $1 \backslash 1-2$ & I\A & $x$ & $x$ \\
\hline \multicolumn{7}{|l|}{ Tabebuia lepidophylla } \\
\hline 1. Santos e Miller (1992) & $4-5 *$ & $x$ & 1 & A & & $x$ \\
\hline \multicolumn{7}{|l|}{ Tabebuia lepidota } \\
\hline 1. Santos e Miller (1992) & $4-5 *$ & $x$ & $1-2$ & 1 & $x$ & \\
\hline \multicolumn{7}{|l|}{ Tabebuia leptoneura } \\
\hline 1. Santos e Miller (1992) & $5-6 *$ & $x$ & $1-2$ & 1 & $x$ & \\
\hline \multicolumn{7}{|l|}{ Tabebuia maxonii } \\
\hline 1. Santos e Miller (1992) & $4 *$ & $x$ & 2 & A & & $x$ \\
\hline \multicolumn{7}{|l|}{ Tabebuia myrtifolia } \\
\hline 1. Santos e Miller (1992) & $4 *$ & $x$ & 1 & A & & $x$ \\
\hline \multicolumn{7}{|l|}{ Tabebuia nodosa } \\
\hline 1. Santos e Miller (1992) & $6-8 *$ & & $2-3$ & $N$ & & $x$ \\
\hline \multicolumn{7}{|l|}{ Tabebuia obtusifolia } \\
\hline 1. Pace et al. (2015) & & & 2 & $?$ & $x$ & \\
\hline 2. Santos e Miller (1992) & 4 & $x$ & 1 & 1 & $x$ & \\
\hline 3. Alves e Angyalossy- Alfonso (2002) & & & 2 & & $x$ & \\
\hline 4. Mainieri (1983) & & & & A & & \\
\hline 5. Insidewood & & & $1-2$ & A & $x$ & \\
\hline \multicolumn{7}{|l|}{ Tabebuia orinocensis } \\
\hline 1. Santos e Miller (1992) & $4-5 *$ & $x$ & $1-2$ & A & & $x$ \\
\hline 2. Insidewood & & $x$ & 1 & A & $x$ & \\
\hline \multicolumn{7}{|l|}{ Tabebuia pallida } \\
\hline 1. Gasson e Dobbins (1991) & 3,3 & $x$ & $1-2$ & $\mathrm{~N}$ & $x$ & \\
\hline 2. Insidewood & & & $1-2$ & $\mathrm{~N}$ & & \\
\hline \multicolumn{7}{|l|}{ Tabebuia revoluta } \\
\hline 1. Santos e Miller (1992) & 4 & $x$ & 1 & A & & $x$ \\
\hline \multicolumn{7}{|l|}{ Tabebuia rigida } \\
\hline 1. Pace et al. $(2013 ; 2015)$ & & & 2 & $\mathrm{~N}$ & $x$ & \\
\hline 2. Alves e Angyalossy- Alfonso (2002) & & & 2 & & $x$ & \\
\hline 3. Mainieri (1983) & & & & $N$ & & \\
\hline 4. Insidewood & & & $1-2$ & $\mathrm{~N}$ & $x$ & \\
\hline \multicolumn{7}{|l|}{ Tabebuia rosea } \\
\hline 1. León (2012) & 4,1 & $x$ & $1-2$ & $\mathrm{~N}$ & $x$ & \\
\hline 2. León (2015) & $3,7-6,2$ & $x$ & $1-3$ & $\mathrm{~N}$ & $x$ & $x$ \\
\hline 3. Reis e Callado (2013) & & & $1-4$ & $\mathrm{~N}$ & & \\
\hline 4. Yajure (2014) & 4,7 & $x$ & $1-3$ & $N$ & $x$ & \\
\hline 5. León (2007) & 4,9 & $x$ & $1-3$ & $\mathrm{~N}$ & $x$ & \\
\hline 6. Santos e Miller (1992) & $5-7^{*}$ & $x$ & $1-4$ & $N \backslash I \backslash A$ & $x$ & \\
\hline 7. Gasson e Dobbins (1991) & & & $1-3$ & $N$ & $x$ & \\
\hline 8. León (2014) & $3,7-6,2$ & $x$ & $1-3$ & $\mathrm{~N}$ & $x$ & \\
\hline 9. Insidewood & & $x$ & $1-3$ & $N \backslash I$ & $x$ & \\
\hline \multicolumn{7}{|l|}{ Tabebuia roseoalba } \\
\hline 1. Pace et al. $(2013 ; 2015)$ & & & $1 \backslash 1-2$ & ? & $x$ & \\
\hline 2. Santos e Miller (1992) & $3-4$ & $x$ & $1-3$ & 1 & $x$ & \\
\hline 3. Gasson e Dobbins (1991) & 4,1 & $x$ & $1-2$ & $\mathrm{~N}$ & $x$ & \\
\hline 4. Alves e Angyalossy- Alfonso (2002) & & & 2 & & $x$ & \\
\hline 5. Insidewood & & & $1-2$ & $\mathbf{I}$ & $x$ & \\
\hline \multicolumn{7}{|l|}{ Tabebuia sauvallei } \\
\hline 1. Santos e Miller (1992) & $4-5 *$ & $x$ & $2-3$ & I & $x$ & \\
\hline Tabebuia stenocalyx & & & & & & \\
\hline
\end{tabular}




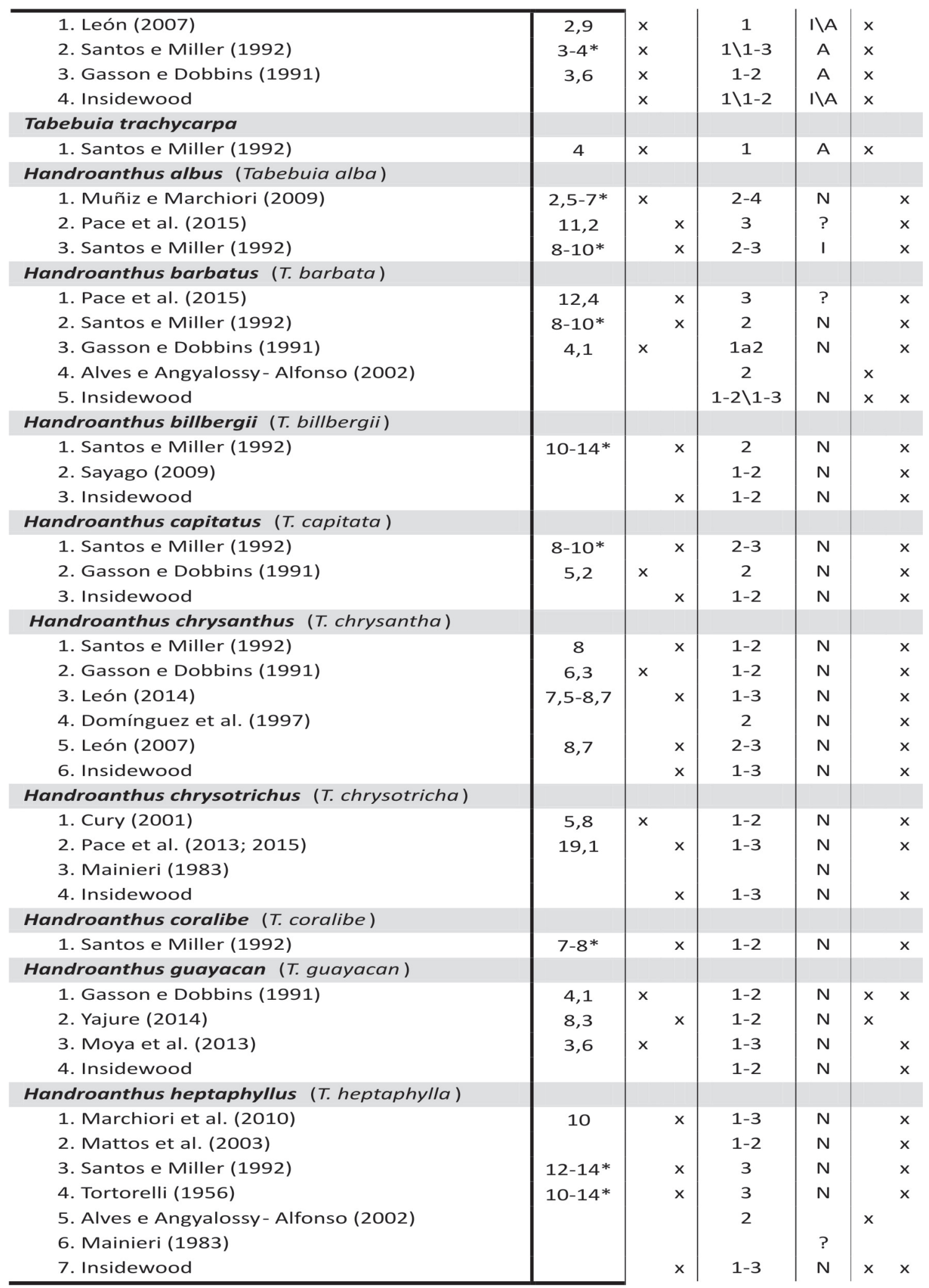




\begin{tabular}{|c|c|c|c|c|c|c|c|}
\hline \multicolumn{8}{|c|}{ Handroanthus impetiginosus ( $T$. impetiginosa) } \\
\hline 1. Mattos et al. (2003) & & & & $1-3$ & 1 & & $x$ \\
\hline 2. León (2007) & 9,9 & & $x$ & 2 & $\mathbf{N}$ & & $x$ \\
\hline 3. Trevizor (2011) & 10,5 & & $x$ & $1-3$ & $\mathbf{N}$ & & $x$ \\
\hline 4. Albuquerque (2012) & 7 & $x$ & & $1-3$ & $\mathbf{N}$ & $x$ & $x$ \\
\hline 5. Pace et al. (2015) & 12,2 & & $x$ & 3 & ? & & $\mathrm{x}$ \\
\hline 6. Santos e Miller (1992) & 12 & & $x$ & $2-3$ & $\mathbf{N}$ & & $x$ \\
\hline 7. Gasson e Dobbins (1991) & 7,1 & & $x$ & 2 & $\mathbf{N}$ & & $\mathrm{x}$ \\
\hline 8. Alves e Angyalossy- Alfonso (2002) & & & & $2 \backslash 3-4$ & & & $x$ \\
\hline 9. Coradin et al. (2010) & & & & & 1 & & \\
\hline 10. Mainieri (1983) & & & & & ? & & \\
\hline 11. Insidewood & & & $x$ & $1-3 \backslash 1-4$ & $\mathbf{N}$ & $x$ & $x$ \\
\hline \multicolumn{8}{|l|}{ Handroanthus incanus ( $T$. incana) } \\
\hline 1. Trevizor (2011) & 10,5 & & $x$ & $1-3$ & $\mathbf{N}$ & & $\mathrm{x}$ \\
\hline 2. Albuquerque (2012) & 4 & $x$ & & $2-4$ & $\mathbf{N}$ & $x$ & \\
\hline 3. Mainieri (1983) & & & & & $\mathbf{N}$ & & \\
\hline 3. Coradin et al. (2010) & & & & & 1 & & \\
\hline \multicolumn{8}{|l|}{ Handroanthus ochraceus (T. ochracea) } \\
\hline 1. Mattos et al. (2003) & & & & $1-2$ & $\mathbf{N}$ & & $x$ \\
\hline 2. Yajure (2014) & 9,7 & & $x$ & $1-3$ & $\mathbf{N}$ & & $x$ \\
\hline 3. Santos e Miller (1992) & $10-12 *$ & & $x$ & $2-3$ & $\mathbf{N}$ & & $\mathrm{x}$ \\
\hline 4. Coradin et al. (2010) & & & & & 1 & & \\
\hline 5. Mainieri (1983) & & & & & $\mathbf{N}$ & & \\
\hline 6. Insidewood & & & & $1-3$ & $\mathbf{N}$ & & $\mathrm{x}$ \\
\hline \multicolumn{8}{|l|}{ Handroanthus pulcherrimus (T. pulcherrima) } \\
\hline 1. Marchiori (2009) & 9,5 & & $x$ & $1-3$ & $\mathbf{N}$ & & $\mathrm{x}$ \\
\hline 2. Santos e Miller (1992) & $10-12 *$ & & $x$ & $2-3$ & $\mathbf{N}$ & & $x$ \\
\hline \multicolumn{8}{|l|}{ Handroanthus serratifolius ( $T$. serratifolia) } \\
\hline 1. León (2015) & $8,7-10$ & & $x$ & $2-3$ & $\mathbf{N}$ & & $\mathrm{x}$ \\
\hline 2. León (2007) & 9,7 & & $x$ & $1-3$ & $\mathbf{N}$ & & $x$ \\
\hline 3. Paula et al. (2000) & & & & $2-3$ & $\mathbf{N}$ & & $x$ \\
\hline 4. Trevizor (2011) & 6,4 & $x$ & & $1-3$ & $N$ & & $x$ \\
\hline 5. Albuquerque (2012) & 5 & $x$ & & $2-3$ & $\mathbf{N}$ & $x$ & $x$ \\
\hline 6. Pace et al. (2015) & 11,3 & & $x$ & 2 & $?$ & & $x$ \\
\hline 7. Santos e Miller (1992) & $10-12 *$ & & $x$ & $2-3$ & $\mathbf{N}$ & & $x$ \\
\hline 8. Gasson e Dobbins (1991) & 4,4 & $x$ & & $1-2$ & $\mathbf{N}$ & & $x$ \\
\hline 9. Miller e Detienne (2001) & $10-12$ & & $x$ & $2-3$ & $\mathbf{N}$ & & $x$ \\
\hline 10. Alves e Angyalossy- Alfonso (2002) & & & & 2 & & & $x$ \\
\hline 11. León (2014) & $8,7-11,2$ & & $x$ & $1-3$ & $\mathbf{N}$ & & $x$ \\
\hline 12. Coradin et al. (2010) & & & & & 1 & & \\
\hline 13. Mainieri (1983) & & & & & $\mathbf{N}$ & & \\
\hline 14. Insidewood & & & & $1-2 \backslash 1-3$ & $N \backslash I$ & & $x$ \\
\hline \multicolumn{8}{|l|}{ Handroanthus uleanus ( $T$. uleana) } \\
\hline 1. Santos e Miller (1992) & 12 & & $x$ & $2-3$ & $\mathbf{N}$ & & $x$ \\
\hline \multicolumn{8}{|l|}{ Handroanthus umbellatus (T. umbellata) } \\
\hline 1. Marchiori et al. (2010) & $5-10 *$ & & & $1-2$ & $\mathbf{N}$ & $x$ & \\
\hline 2. Barros e Callado (1997) & 7,3 & & $x$ & $1-2$ & $\mathrm{~N}$ & $x$ & \\
\hline 3. Mainieri (1983) & & & & & $?$ & & \\
\hline 4. Coradin et al. (2010) & & & & & $N \backslash I$ & & \\
\hline \multicolumn{8}{|l|}{ Roseodendron chryseum ( $T$. chrysea) } \\
\hline 1. Santos e Miller (1992) & $6-7^{*}$ & $x$ & & $2-3$ & I\A & & \\
\hline
\end{tabular}




\begin{tabular}{l|c|c|c|c|c}
\hline 2. Gasson e Dobbins (1991) & 4,1 & $\mathrm{x}$ & $2-4$ & $\mathrm{~A}$ & $\mathrm{x}$ \\
3. León (2007) & 5,9 & $\mathrm{x}$ & $2-3$ & $\mathrm{~N}$ & $\mathrm{x}$ \\
4. Insidewood & & & $1-3$ & $\mathrm{~A}$ & \\
Roseodendron donnell-smithii (T. donnell-smithii) & & & & & \\
1. Santos e Miller (1992) & $5-8^{*}$ & & $3-5$ & I\A & \\
2. Gasson e Dobbins (1991) & 5 & $\mathrm{x}$ & $2-5$ & $\mathrm{~A}$ & $\mathrm{x}$ \\
3. Pace et al (2013; 2015) & 5,1 & $\mathrm{x}$ & $2-4$ & ?\A & \\
4. Insidewood & & & $1-4 \backslash>4$ & I\A & $\mathrm{x}$ \\
\hline
\end{tabular}

Onde: $\mathrm{PIV}=$ pontoações intervasculares $(\varnothing=$ diâmetro em $\mu \mathrm{m} ; \mathrm{dp}=$ diminutas a pequenas; $\mathrm{mg}=\mathrm{médias}$ a grandes; *=valores mínimos e máximos). $\mathrm{RL}=$ raios largura (cels= células); $\mathrm{RE}=$ raios estratificação $(\mathrm{I}=$ irregular; $\mathrm{N}=$ normal; $\mathrm{A}=$ ausente; ?= presente, mas indefinida); $\mathrm{EPF}=$ espessura da parede de fibras (fe= finas a espessas; me= espessas a muito espessas). Os nomes entre parênteses são as atuais sinonímias.

desenvolvimento ou a capacidade de desenvolvimento de pontoações intervasculares de maior diâmetro é, na prática, uma particularidade de Handroanthus, já que entre todas as espécies investigadas de Tabebuia há apenas uma que apresenta pontoações médias a grandes $(>7 \mu \mathrm{m})$. Pela consistência com que ocorre, este é, sem dúvida, o caráter anatômico mais importante para a taxonomia dos gêneros analisados.

No caso da estratificação dos raios, três estados de caráter podem ser observados nas madeiras: normal, irregular e ausente (Figura $1,2)$. Essa variação pode ocorrer tanto entre espécies quanto entre diferentes indivíduos de uma mesma espécie, sendo especialmente notável em certos representantes de Tabebuia, como Tabebuia aurea, Tabebuia heterophylla, Tabebuia insignis e Tabebuia rosea, que podem apresentar os três estados de caráter possíveis. A ocorrência de estratificação normal de raios é generalizada em Handroanthus, mas não é exclusiva deste gênero, uma vez que várias espécies de Tabebuia também podem apresentar estrutura estratificada bem definida (Tabela 1; Figura 1). A ausência de estratificação, por outro lado, embora nem sempre observada, é uma possibilidade estrutural exclusiva de Tabebuia. Já a estratificação irregular pode ocorrer em ambos os gêneros, mas é bem mais frequente em espécies de Tabebuia. Sobre esse assunto, cabe, ainda, um último comentário. Se os raios são estratificados ou não é algo relativamente fácil de ser observado e registrado. A distinção entre estratificação normal e irregular, importante para a separação dos gêneros, é, todavia, um detalhe anatômico não tão óbvio, podendo ser facilmente ignorado, o que pode levar a erros, já que se trata de um trabalho de revisão.

Quanto à largura dos raios em número de células, este também se mostrou um caráter bastante variável, inclusive dentro de uma mesma espécie. No entanto, algumas diferenças podem ser reconhecidas. No gênero Tabebuia, predominam as espécies com raios de 1-2 células de largura, ao passo que em Handroanthus são bem mais frequentes, quase uma unanimidade, as espécies com raios de até 3 células de largura, o que equivale a dizer que neste gênero os raios são geralmente um pouco mais largos em relação àquele. Outra característica importante que ajuda a estabelecer as diferenças na largura dos raios entre os dois grupos é a ocorrência de raios exclusivamente unisseriados, a qual é observada apenas em certas espécies de Tabebuia, embora nem sempre de maneira muito consistente (Tabela 1). Em Handroanthus não são observadas espécies com raios exclusivamente unisserridos, nem mesmo de forma ocasional. 


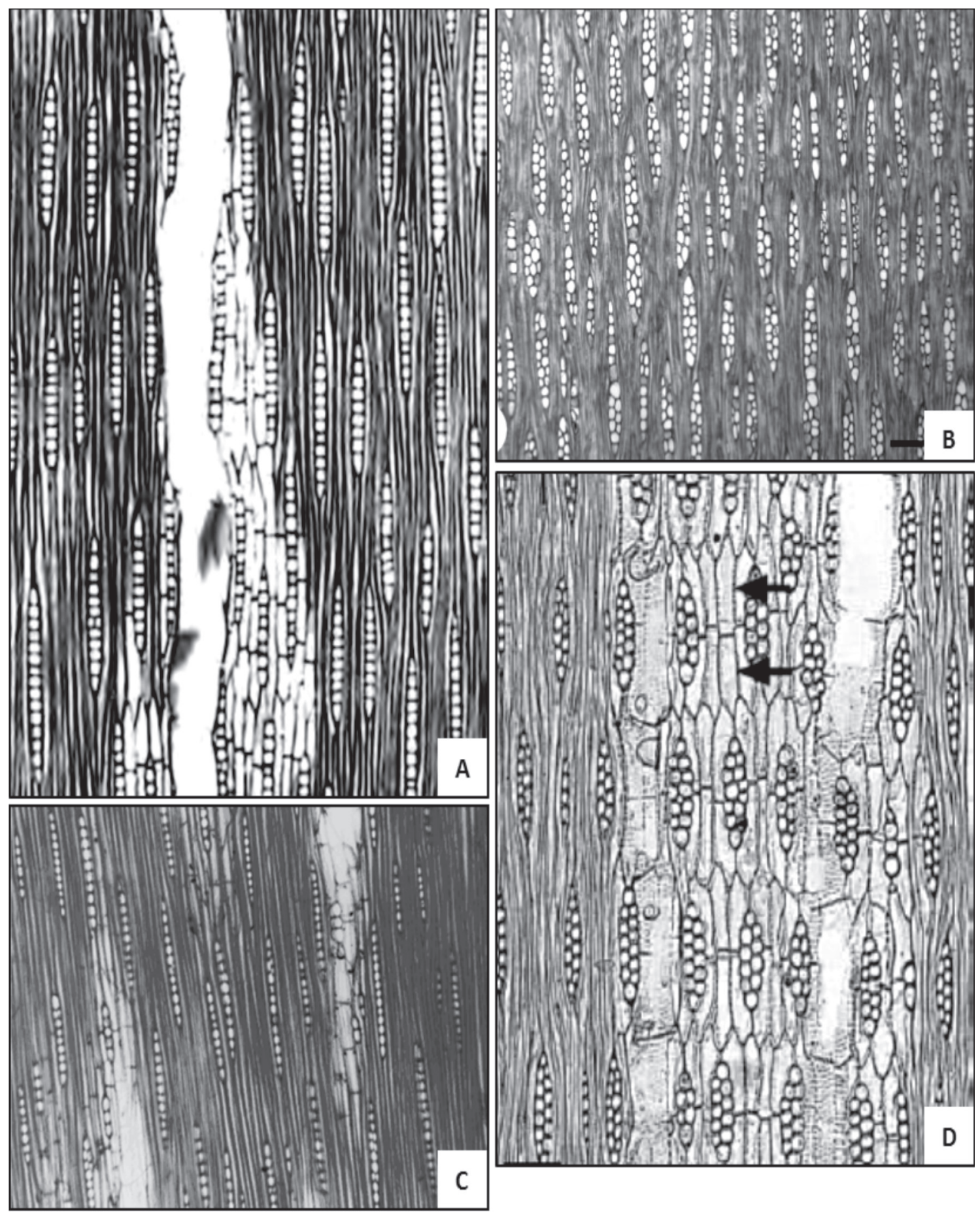

FIGURA 1 - Tipos de estratificação em espécies de Tabebuia. A e B, estratificação irregular em Tabebuia insignis (foto: Miller e Detienne, 2001) e Tabebuia aurea (Dória, 2014), respectivamente. C, ausência de estratificação em Tabebuia stenocalyx (León, 2007). D, estratificação normal em Tabebuia rigida (Pace e Angyalossy, 2013). As setas em D indicam uma série de parênquima axial. 
À exemplo da estratificação, a largura dos raios mostrou-se notavelmente incerta em algumas espécies de Tabebuia. É o caso de Tabebuia aurea, Tabebuida cassinoides, Tabebuia fluviatilis, Tabebuia roseoalba e Tabebuia stenocalyx, entre outras, que tem raios desde exclusivamente unisseriados até multisseriados, com 2 ou 3 células de largura (Tabela 1). Santos e Miller (1992) já haviam chamado a atenção para a variabilidade nos raios de algumas espécies de Tabebuia, posicionandoas, inclusive, em um grupo separado (Grupo III). Apesar da separação inicial, todas fazem parte do mesmo gênero, atualmente, e, pelo que consta em estudos filogenéticos recentes de Tabebuia, que incluem ao menos algumas delas; essa é a interpretação mais correta (Grose e Olmstead, 2007a). Não obstante, alguns estudos já demonstraram que pode haver uma considerável variação nas características anatômicas dos raios e outros tipos celulares de certas espécies de Bignoniaceae, como Tabebuia cassinoides (Carrera, 2008) e Tabebuia aurea (Doria, 2014), influenciadas pelo ambiente.

No tocante à espessura da parede das fibras, cabe salientar, de início, que a mesma pode ser considerada um dos aspectos estruturais mais antigos na segregação dos Ipês, tendo em vista a influência direta no peso ou densidade da madeira, que foi um dos critérios utilizados por Record e Hess (1943) para a definição do grupo "lapacho". Este caráter anatômico, como os anteriores, também exibe variação entre espécies e dentro de uma mesma espécie; mesmo assim, é possível identificar tendências divergentes nos novos gêneros, em concordância com o referido na literatura (Record Hess, 1943; Santos e Miller, 1992; Pace et al, 2015).

Quase todas as espécies de Handroanthus apresentam fibras de paredes espessas a muito espessas, ao passo que aquelas com fibras de paredes finas a espessas são bem menos frequentes. Por outro lado, a grande maioria das espécies de Tabebuia têm fibras finas a espessas, sendo pouco numerosas as que apresentam paredes espessas a muito espessas, ainda mais de maneira consistente (Tabela 1). Pode-se dizer, em síntese, que as espécies de Handroanthus tendem a desenvolver fibras de paredes mais espessas, que resultam, geralmente, na formação de madeiras mais pesadas, enquanto que os representantes de Tabebuia tendem a apresentar fibras de paredes mais finas, resultando, presumivelmente, em madeiras mais leves. Há, no entanto, exceções, e algumas espécies, tanto em Handroanthus quanto em Tabebuia, podem apresentar, inclusive, os dois estados de caráter (ex. Tabebuia aurea, Tabebuia rosea, Handroanthus heptaphyllus e Handroanthus impetiginosus).

Em relação ao gênero Roseodendron, observou-se que, exceto pela largura dos raios de Roseodendron donnell-smithii, a qual atinge, comumente, 4-5 células, as espécies apresentam características anatômicas que estão dentro das possibilidades estruturais de Tabebuia (estratificação ausente ou irregular, fibras de paredes finas e pontoações intervasculares diminutas a pequenas). O curioso, neste caso, é que em análises filogenéticas recentes, Roseodendron donnell-smithii aparece mais próxima a Handroanthus do que a Tabebuia, ao contrário do que sugere a sua anatomia. Devido à peculiaridade dos raios, é plenamente justificável a segregação de Roseodendron donnell-smithii em um gênero separado, concordando-se, neste aspecto, com diversos estudos que apontam nesta mesma direção (Grose e Olmstead, 2007a,b; Pace et al., 2015). A outra integrante do gênero, Roseodendron chryseum, não apresenta a mesma peculiaridade nos raios, que separa Roseodendron donnellsmithii das demais; ao contrário, a sua estrutura anatômica é perfeitamente compatível com Tabebuia, inclusive pelo tamanho das pontoações intervasculares (Tabela 1).

Uma das principais razões pela qual Roseodendron chryseum não fora anteriormente incluída no grupo de espécies que hoje forma o gênero Tabebuia é a presença de lapachol e tiloses nos vasos (Santos e Miller, 1992). Se a ocorrência de lapachol em Roseodendron 
chryseum justifica a sua separação de Tabebuia, também serve, por conseguinte, para separá-la de Roseodendron donnell-smithii, a qual não possui conteúdos dessa natureza nos vasos. A ocorrência de tiloses, por sua vez, é uma das únicas características anatômicas não observadas nos outros dois gêneros, que poderia justificar a união de Roseodendron donnellsmithii e Roseodendron chryseum. No entanto, se for considerado que apenas o desenvolvimento de tiloses no interior dos vasos é suficiente para caracterizar Roseodendron, seria necessário incluir Tabebuia fluviatilis neste gênero, uma vez que a mesma também conta com esse detalhe anatômico. Por estas razões e, sobretudo, em virtude da ausência de afinidade estrutural, não parece fazer muito sentido, do ponto de vista da anatomia da madeira, a inclusão de Roseodendron donnell-smithii e Roseodendron chryseum no mesmo grupo taxonômico.

Vale lembrar que as duas espécies de Roseodendron, assim como Tabebuia fluviatilis e Tabebuia nodosa, não foram incluídas por Santos e Miller (1992) em nenhum dos grupos atualmente correspondentes aos gêneros Tabebuia e Handroanthus, sob o argumento de não se enquadrarem bem nos mesmos e/ou por apresentarem particularidades estruturais que justificam tal fato. Exceto por Roseodendron chryseum, que apresenta características anatômicas compatíveis com Tabebuia (desconsiderando a ocorrência de lapachol, que não foi analisada no trabalho), os resultados deste estudo concordam com o referido por Santos e Miller (1992). Tabebuia fluviatilis apresenta pontoações médias a grandes, sendo a única espécie investigada de Tabebuia com pontoações que excedem o limite estabelecido para o referido gênero. Roseodendron donnellsmithii, como anteriormente discutido, singulariza-se pela largura dos raios. Tabebuia nodosa reúne uma combinação de características (raios regularmente estratificados, com 2-3 células de largura e fibras de paredes muito espessas) pouco comum em espécies de
Tabebuia, aspectos que a aproximam de Handroanthus (Tabela 1). Infelizmente, este foi um dos poucos casos em que não foi possível, por questões metodológicas, utilizar os valores referidos para o tamanho das pontoações. Pela importância taxonômica deste caráter na discussão dos gêneros, não há como fazer maiores considerações sobre o posicionamento de Tabebuia nodosa, ainda mais sendo este, justamente, um dos aspectos que tornam a espécie atípica, segundo Santos e Miller (1992). A distribuição definitiva desta e das demais espécies com estrutura atípica nos gêneros, depende, como salientado Pace et al. (2015), da realização de estudos filogenéticos adicionais, ainda mais levando-se em conta que, das quatros espécies, Roseodendron donnell-smithii é a única que foi incluída em análises moleculares do gênero Tabebuia (Grose e Olmstead, 2007a).

A análise da estrutura anatômica das 43 espécies de Tabebuia (latu sensu) presentemente investigadas, depõe, favoravelmente, à segregação dos três novos gêneros, conforme sugerido em estudos taxonômicos recentes (Grose e Olmstead, 2007a,b). Apesar de não haver uma característica ou um conjunto de características anatômicas comuns e exclusivas ao grupo de espécies de cada um dos gêneros, é inegável, do modo como estão atualmente circunscritos, que existem tendências estruturais diferentes que justificam a sua separação. No entanto, algumas dúvidas persistem e estudos adicionais mostram-se necessários, até que se possa estabelecer, com mais segurança, os limites genéricos e a correto posicionamento de algumas espécies consideradas anômalas (Santos e Miller, 1992).

As diferenças observadas no tamanho das pontoações intervasculares, na estratificação e largura dos raios, bem como na espessura da parede das fibras confirmam, mais uma vez, o valor taxonômico destas características, reconhecidas, aliás, ao longo do tempo em estudos anatômicos de Tabebuia (Record e Hess, 1940; Record e Hess, 1943; Santos e Miller, 1992; Pace et al., 2015). Também ficou 

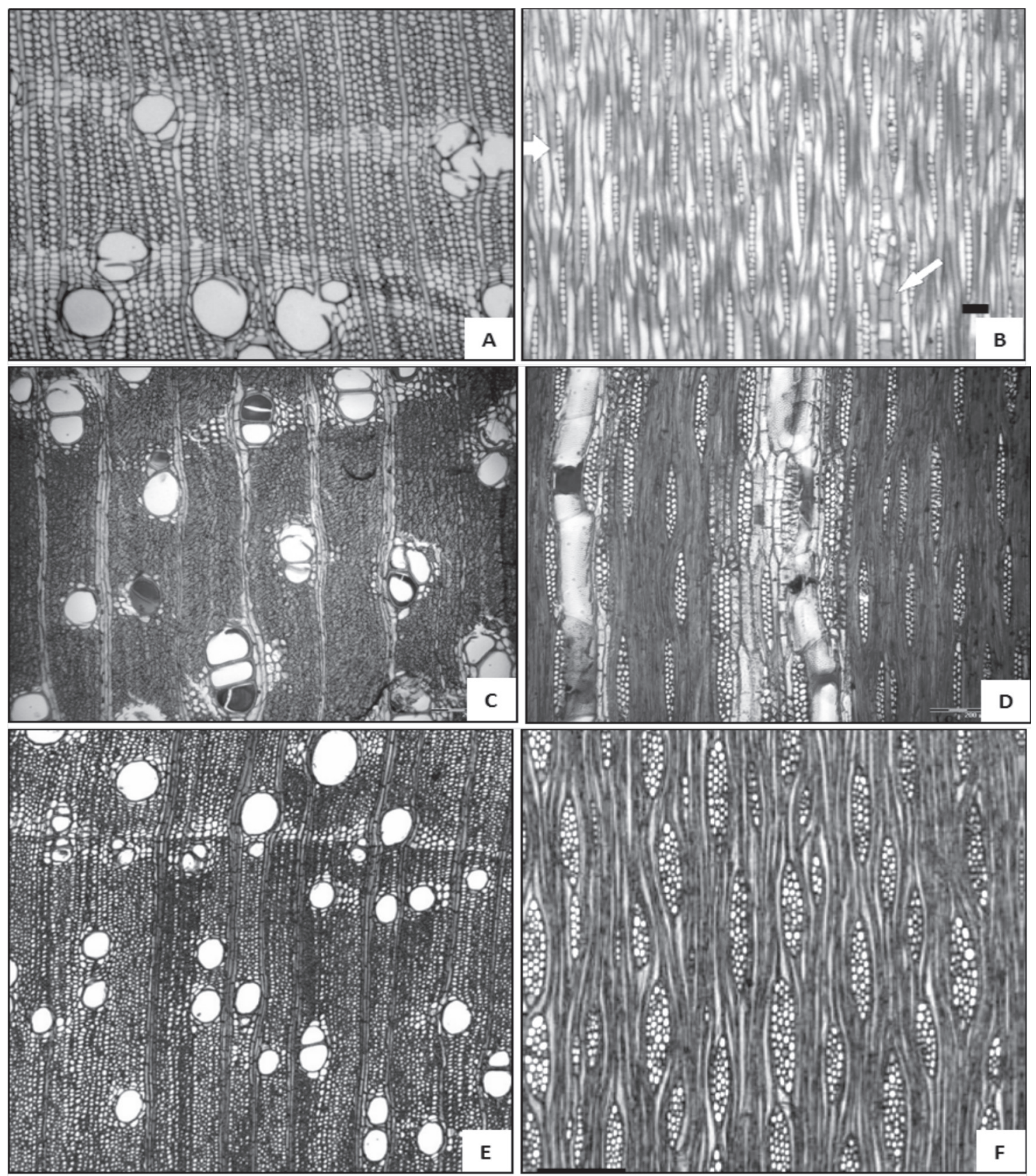

FIGURA 2 - A anatomia da madeira nos três gêneros. A e B, Tabebuia cassinoides evidenciando fibras finas a espessas e raios não estratificados, comuns em espécies de Tabebuia. As setas em B estão indicando raios unisseriados e séries de parênquima axial (fotos: Carrera, 2008). C e D, Handroanthus heptaphyllus mostrando fibras muito espessas e raios com estratificação normal, característicos de Handroanthus (fotos: Marchiori et al., 2010). E e F, Roseodendron donnellsmithii com fibras de paredes finas a espessas e raios largos, não estratificados (fotos: E, Marcelo Pace, Insidewood; F, Pace et al., 2015). 
confirmado, mais uma vez, o acerto, ainda que parcial, de Santos e Miller (1992) ao separar as espécies de Tabebuia em grupos de afinidade anatômica. O grupo I correspondendo perfeitamente a Handroanthus, e os demais grupos e espécies, com exceção das que hoje formam o gênero Roseodendron, correspondendo à Tabebuia. Embora para a maioria das espécies tenha sido possível reunir mais do que uma amostra/referência anatômica, em alguns casos isso infelizmente não foi possível, o que representa, sem dúvida, uma limitação ao estudo, dada a grande variabilidade inter e intraespecífica observada nos gêneros. Além disso, este trabalho focou na análise de apenas algumas características microscópicas tradicionalmente utilizadas e mais disponíveis na literatura, sendo recomendável o exame aprofundado de outros caracteres anatômicos potencialmente úteis à separação e identificação dos gêneros, como a presença de conteúdos nos vasos, altura dos raios e características do parênquima axial.

\section{REFERÊNCIAS BIBLIOGRÁFICAS}

ALBUQUERQUE, A.R. Anatomia comparada do lenho e do carvão aplicada na identificação de 75 espécies da Floresta Amazônica no estado do Pará, Brasil. 2012. 249 f. Dissertação de Mestrado - Escola Superior de Agricultura Luiz de Queiroz (ESALQ), Piracicaba, 2012.

ALVES, E.S.; ANGYALOSSY-ALFONSO, V. Ecological trends in the wood anatomy of some brazilian species 2 . Axial parenchyma, rays and fibers. IAWA Journal, v. 23, n. 4, p. 391-418, 2002.

BARROS, C.F.; CALLADO, C.H. Madeiras da Mata Atlântica. Anatomia do lenho de espécies ocorrentes nos remanescentes florestais do estado do Rio de Janeiro, Brasil. Rio de Janeiro: Instituto de Pesquisas Jardim Botânico do Rio de Janeiro, 1997. 86 p.

CANDOLLE, A.P. Revue sommaire de la famille des Bignoniacées. Genève: Bibliotheque Universelle de Genève. 1838. 20 p.

CARRERA, U.S. Variação intra-específica na anatomia da folha e do lenho de Tabebuia cassinoides (Lam.) DC. (Bignoniaceae) em ambientes distintos. 2008. 46 f. Dissertação de Mestrado - Instituto de Pesquisa Jardim Botânico do Rio de Janeiro, Rio de Janeiro, 2008.
CORADIN, V.T.R.; CAMARGOS, J.A.A.; PASTORE, T.C.M.; CHRISTO, A.G. Madeiras Comerciais do Brasil: chave interativa de identificação baseada em caracteres gerais e macroscópicos. Serviço Florestal Brasileiro, Laboratório de Produtos Florestais: Brasília, 2010.

CURY, G. Descrição da estrutura anatômica do lenho e sua aplicação na identificação de espécies arbóreas do cerrado e da mata atlântica do estado de São Paulo. 2002. 125 f. Dissertação de Mestrado - Escola Superior de Agricultura Luiz de Queiroz (ESALQ), Piracicaba, 2002.

DOMÍNGUEZ, S.R.; PÉREZ-OLIVEIRA, C.P.; ISAÍAS, A.L. Anatomía de la madera de ocho especies de la selva mediana subperenifolia de Quintana Roo, México. Revista de Biologia Tropical, v. 44(3) /45(1), p. 67-77, 1997.

DÓRIA, L.C. Anatomia comparada do lenho de Tabebuia aurea (Bignoniaceae) e Tocoyena formosa (Rubiaceae) que ocorrem no Cerrado e na Caatinga. 2014. 123 f. Dissertação de Mestrado - Universidade Estadual Paulista (UNESP), Botucatu, 2014.

FARIA, M.C.G. Potencial madeireiro de 22 espécies arbóreas do Cerrado: caracterização anatômica, física e energética. 2016. 103 f. Dissertação de Mestrado - Universidade Federal de Goiás (UFG), Goiânia, 2016.

FLORADOBRASIL. Jardim Botânico do Rio de Janeiro. Disponível em: <http://floradobrasil.jbrj.gov.br/>. Acesso em: 12 Maio de 2017.

GASSON, P.; DOBBINS, D.R. Wood anatomy of the Bignoniaceae, with a comparison of trees and lianas, IAWA Bulletin, v. 12, p. 389-417, 1991.

GENTRY, A.H. Handroanthus (Bignoniaceae): a critique. Taxon, n. 21, p. 113-114, 1972.

GENTRY, A.H. Bignoniaceae. Part II (Tribe Tecome). Flora Neotropica, n. 25 (2), p. 1-370, 1992.

GROSE, S.O.; OLMSTEAD, R.G. Evolution of a charismatic neotropical tree: Molecular phylogeny of Tabebuia s.l. and allied genera (Bignoniaceae). Systematic Botany, v. 32 (3), p. $650-659,2007 \mathrm{a}$.

GROSE, S.O.; OLMSTEAD, R.G. Taxonomic revisions in the polyphyletic genus Tabebuia s.l. (Bignoniaceae). Systematic Botany, v. 32 (3), p. 660-670, 2007b.

INSIDEWOOD. Published on the Internet. < http:// insidewood.lib.ncsu.edu/search>. Acesso em: 08 de Maio de 2017. 2004-onwards.

KUNIYOSHI, Y.S. Aspectos morfo-anatômicos do 
caule, raiz e folha de Tabebuia cassinoides (Lam.) DC. (Bignoniaceae) em diferentes fases sucessionais no litoral do Paraná. 1993. 147 f. Tese de Doutorado - Universidade Federal do Paraná (UFPR), Curitiba, 1993.

LEÓN, H. Anatomía del xilema secundario de diez espécies de la familia Bignoniaceae de Venezuela. Acta Botánica Venezuelica, v. 30 (2), p. 361-384, 2007.

LEÓN, H. Anatomía de la madera de 69 especies de la Reserva Forestal Caparo (Barinas, Venezuela). Revista Forestal Venezolana, v. 56 (2), p. 167 195, 2012.

LEÓN, H. Anatomía de Madera de 108 especies de Venezuela. Pittieria: Serie de Publicaciones Especiales, n. 1, 2014. 267 p.

LEÓN, H. Anatomía de la madera de 87 especies de la Reserva Forestal Ticoporo (Barinas, Venezuela). Pittieria, v. 39, p. 107-169, 2015.

MAINIERI, C. Manual de Identificação das Principais Madeiras Comerciais Brasileiras. São Paulo: PROMOCET, $241 \mathrm{p}$.

MARCHIORI, J.N.C. Estudo anatômico da madeira de Handroanthus pulcherrimus (Sandwith) S. Grose. Balduinia, n.19, 10-13, 2009.

MARCHIORI, J.N.C; MUÑIZ, G.I.B.; SANTOS, S.R. Madeiras do Rio Grande do Sul. 2 Descrição microscópica de 35 espécies nativas. Santa Maria: Anaterra, 2010. 80 p.

MATTOS, J.R. Handroanthus, um novo gênero para os "ipês" do Brasil. Loefgrenia, n. 50, p. 1-4, 1970.

MATTOS, P.P.; TEIXEIRA, L.L.; SEITZ, R.A.; SALIS, S.M.; BOTOSSO, P.C. Anatomia de madeiras do Pantanal Mato-Grossense (características microscópicas). Embrapa Florestas, Colombo, PR. Embrapa Pantanal, Corumbá, PR, 2003. 182 p.

MILLER, R.B.; DÉTIENNE, P. Major Timber Trees of Guyana. Wood anatomy. Tropenbos International, Wageningen, 2001. 218 p.

MOYA, R.; WIEMANN, M.C.; OLIVARES, C. Identification of endangered or threatened Costa Rican tree species by wood anatomy and fluorescence activity. Revista de Biologia Tropical, v. 61(3), p. 1133-1156, 2013.

MUÑIZ, G.I.B.; MARCHIORI, J.N.C. Anatomia da madeira de duas Bignoniáceas da Floresta Estacional de Missiones - Argentina. Balduinia, n.18, 20-25, 2009.

PAULA, J.E.; JUNIOR, F.G.S.; SILVA, A.P.P. Caracterização anatômica de madeiras nativas de matas ciliares do centro-oeste brasileiro. Scientia Forestalis, v. 58, p. 73-89, 2000.

PACE, M.R.; ANGYALOSSY, V. Wood anatomy and evolution: a case study in the Bignoniaceae. International Journal of Plant Science, v. 174 (7), p. 1014-1048, 2013.

PACE, M.R.; LOHMANN, L.G..; OLMSTEAD, R.G.; ANGYALOSSY, V. Wood anatomy of major Bignoniaceae clades. Plant Systematics and Evolution, v. 301, p. 967-995, 2015.

RECORD, S.J.; HESS, R.W. American Timbers of the Family Bignoniaceae. Tropical Woods, n. 63, p. 9-38, 1940.

RECORD, S.J.; HESS, R.W. Timbers of the New World. New Haven: Yale University Press, 1943. $640 \mathrm{p}$.

REIS, M.S.A.; CALLADO, C.H. Anatomia do lenho de Tabebuia rosea (Bertol.) A. DC. (Bignoniaceae). Anais do Congresso Brasileiro de Ciência e Técnologia da Madeira, 2013.

SANTOS, G.; MILLER, R.B. Wood anatomy of Tecomeae. Flora Neotropica, n. 25, p. 336-358, 1992.

SAYAGO, J.C.S. Determinación de las características anatómicas y propiedades físicas de la madera de la especie forestal madero negro o guayacán (Tabebuia bilbergii) de la región de Tumbes. 2009. $162 \mathrm{f}$. Tesis. Curso de Ingeniería Forestal y del Medio Ambiente - Universidad Nacional de Tumbes, Tumbes, Peru, 2009.

TORTORELLI, L.A. Maderas y Bosques Argentinos. Buenos Aires: ACME. 1956. 910 p.

TREVIZOR, T.T. Anatomia comparada do lenho de 64 espécies arbóreas de ocorrência natural na Floresta Tropical Amazônica no estado do Pará. 2011. 214 f. Dissertação de Mestrado - Escola Superior de Agricultura Luiz de Queiroz (ESALQ), Piracicaba, 2011.

YAJURE, P. Identificación de Bignoniaceae de la ciudad de Mérida (Venezuela) através de la anatomía de la madera. Pittieria, v. 38, p. 121134, 2014. 